\title{
The Two-dimensional and Three-dimensional T2 Weighted Imaging-based Radiomic Signatures for the Preoperative Discrimination of Ovarian Borderline Tumors and Epithelial Cancer.
}

\section{Xuefen Liu}

Obstetrics and Gynecology Hospital of Fudan University

Tianping Wang

Obstetrics and Gynecology Hospital of Fudan University

Guofu Zhang

Obstetrics and Gynecology Hospital of Fudan University

Keqin Hua

Obstetrics and Gynecology Hospital of Fudan University

Shaofeng Duan

GE Healthcare

Jun Jin

Obstetrics and Gynecology Hospital of Fudan University

He Zhang ( $\Delta$ 13512162249@139.com )

Obstetrics and Gynecology Hospital of Fudan University

Research article

Keywords: Ovarian neoplasm,Magnetic resonance imaging, Computer-Assisted Diagnosis, Radiomics

Posted Date: August 20th, 2020

DOI: https://doi.org/10.21203/rs.3.rs-57353/v1

License: (a) (i) This work is licensed under a Creative Commons Attribution 4.0 International License.

Read Full License 


\section{Abstract}

Background: Accurate discrimination between ovarian borderline tumors (BOTs) and malignancies with imaging play an important role in management.

Methods: A total of 95 patients with pathologically proven ovarian BOTs and 101 patients with malignancies were retrospectively included in this study. We evaluated the diagnostic performance of the signatures derived from T2WI-based radiomics in their ability to differentiate between BOTs and malignancies and compared the performance differences in the 2D and 3D segmentation models. The least absolute shrinkage and selection operator method (LASSO) was used for radiomics feature selection and machine learning processing.

Results: The radiomics score between BOTs and malignancies in four types of selected T2WI-based radiomics models differed significantly at the statistical level $(p<0.0001)$. For the classification between BOTs and malignant masses, the 2D and 3D coronal T2WI-based radiomics models yielded accuracy values of 0.79 and 0.83 in the testing group, respectively; the 2D and 3D sagittal fat-suppressed (fs) T2WI-based radiomics models yielded an accuracy of 0.78 and 0.99 , respectively.

Conclusion: Our results suggest that T2WI-based radiomic features were highly correlated with ovarian tumor subtype classification. 3D-sagittal MRI radiomics features may help clinicians differentiate ovarian BOTs from malignancies with high accuracy (ACC).

\section{Highlights}

- The T2WI radiomics could achieve a higher accuracy in discriminating ovarian tumors.

- The 3D T2WI-based radiomics model showed the better performance than the 2D did.

- The 3D sagittal fat-suppressed T2WI radiomics showed the best performance.

\section{Background}

Ovarian borderline tumors (BOTs) account for approximately $10-15 \%$ of epithelial ovarian tumors, with an annual prevalence of 1.8-4.8/100,000 women worldwide (1). Compared with other ovarian malignant tumors, ovarian BOTs often occur in young patients with early-stage disease, and patients have a good prognosis with fertility-sparing conservative treatments $(2,3)$. Therefore, preoperative identification of patients with ovarian lesions suspected of being BOTs may be helpful in their management.

Magnetic resonance imaging (MRI) has many advantages in determining the etiology of ovarian masses and is widely used in clinical centers (4). MRI has high diagnostic performance in differentiating between ovarian benign tumors and malignant tumors (5-9). Considering the ability to discriminate BOTs from malignant epithelial ovarian tumors, conventional MRI varies with a sensitivity of $58 \%$ to $100 \%$ and a specificity of $61 \%$ to $100 \%$, respectively $(7,10-13)$. Functional MRI scans (dynamic contrast-enhanced MRI, diffusion-weighted imaging(DWI), MR spectroscopy(MRS), etc.) showed a higher ability to 
distinguish a BOT from ovarian epithelial cancer than conventional MRI, such as T1-weighted imaging (T1WI) and T2WI, as shown in recently published studies $(11,12)$. However, given that functional MRI acquisition is not routinely used in clinical scenarios, the scanning parameters are not presently standardized universally and may change across MRI machines or institutions. Gross morphological characteristic imaging features appreciated on T1WI and T2WI still have better applicability in the differentiation of BOTs from other malignancies.

As a research hotspot, radiomics is defined as a new 'data-driven' approach for extracting large sets of quantitative signatures from radiological images and shows its potential application in medicine $(14,15)$. MR-based radiomic signatures has been shown to help to categorize tumor subtypes and assess tumor presence, spread, recurrence or response to treatment in female cancer patients (16-21). To date, there have been limited MRI radiomics studies concerning ovarian BOT and epithelial cancer categorization. The purpose of this research was two-fold: first, we planned to evaluate the diagnostic performance of the MRI radiomics model in discriminating ovarian BOTs from malignancies; second, we sought to clarify whether 3D MR-based radiomic signatures (of the whole lesion) could show better discriminative performance than $2 \mathrm{D}$ radiomic signatures (of the maximum lesion) could in the same study sample.

\section{Methods}

\section{Patients}

Our institutional review board (Gynecological and Obstetric Hospital, School of Medicine, Fudan University, Shanghai, China) approved this retrospective study, and the requirement for informed consent was waived for all participants. From January 2014 to December 2017, 438 consecutive patients with clinically suspected gynecological diseases were retrospectively retrieved from our institutional picture archiving and communication system (PACS, GE). The inclusion criteria were as follows: 1) patients with no previous pelvic surgery; 2) patients with no previous gynecological disease history; and 3) patients who had MRI examinations performed at our institution before pelvic or laparoscopic surgery. A total of 91 patients (average age, $39.8 \pm 14.9$ years) with pathologically proven ovarian borderline tumors and 105 patients with ovarian epithelial malignancies (average age, $51.9 \pm 12.1$ years) were selected as the study sample for signature selection (Table 1). The information on pathological type, immunohistological staining results, and laboratory tests were collected through a hospital information system.

\section{MR image acquisition and lesion segmentation and radiomics feature selection}

MRI was performed using a 1.5-T MR system (Magnetom Avanto, Siemens) with a phased-array coil. The routine MRI protocols used to assess pelvic masses included axial turbo spin-echo (TSE)-T1WI, coronal TSE T2WI, and axial/sagittal TSE fat-suppressed T2WI (fs-T2WI). The detailed MRI acquisition parameters are listed in Supplementary Table 1. All lesion segmentation was performed by an 
experienced radiologist (H.Z.). The maximum lesion segmentation on MRI was manually outlined using ITK-SNAP software (ITK-SNAP, version 3.4.0, www.itksnap.org) (Figure 1). Two segmentation methods were used in this study: maximum lesion segmentation (2D) and whole-lesion segmentation (3D) on both sagittal fs-T2W images and coronal T2W images. In 2D segmentation model, we chose one slice with the largest lesion diameter in two protocols as the premium picture for segmenting the whole lesion. In 3D segmentation model, the entire lesion from both protocols was outlined and segmented slice by slice. After the tumor segmentation process, MR-based radiomics signatures were extracted from 2D/3D sagittal fs-T2W and 2D/3D coronal T2W images using Analysis Kit software(version 3.0.0, GE Healthcare) on a personal computer (Figure 1).

\section{Image feature extraction and selection}

A total of 396 radiomics features from the volume of interest were extracted automatically using in-house software (Analysis Kit, version 3.0.0, GE Healthcare). Thereafter, the whole dataset was randomly divided into two parts: a training cohort and a testing cohort. The radiomics score (Rad-score)-based signatures were constructed with the LASSO method, which was used to select the most useful prognostic features in the training data set. A Rad-score was computed for each patient through a linear combination of selected features weighted by their respective coefficients. These Rad-scores were first assessed in the training data set and then validated in the testing data set.

\section{Statistical analysis}

First, two-sample t-tests were performed to compare MR-based signature values between ovarian BOT and ovarian cancer. Next, the sensitivity (SEN), specificity (SPE), positive predictive value (PPV), and negative predictive value (NPV) were calculated when the performance of the two methods was evaluated for their ability to identify ovarian malignancies. Additionally, receiver operating characteristic (ROC) curve analysis was performed to evaluate various MR-based signature diagnostic values in discriminating BOTs from malignancies. A value of $p<0.05$ was considered statistically significant.

\section{Results}

\section{Clinical characteristics in both the training and testing data sets}

In this study, we included 91 ovarian borderline tumors and 105 ovarian malignancies (83 high-garde serous epithelial carcinomas, 7 mucinous carcinomas, 4 mixed carcinomas, 5 clear cell type carcinomas, 3 endometrioid carcinomas and 3 low-grade carcinomas, Table 1). There was no statistically significant difference found between the training and the validation data set in either clinical characteristics or pathological subtypes (Table 2). 
Table 1

The summary of the pathological types and numbers of the selected samples.

\begin{tabular}{|lll|}
\hline Pathological type & Numbers & Age(yrs.)* \\
\hline Ovarian borderline tumor & 91 & $39.75 \pm 14.85$ \\
\hline Ovary malignancies & 105 & $51.91 \pm 12.05$ \\
\hline Endometroid cancer & 3 & $44.67 \pm 6.02$ \\
\hline Low-grade adenocarcinoma & 3 & $42.33 \pm 19.96$ \\
\hline Clear cell type & 5 & $49.4 \pm 10.33$ \\
\hline High-grade serous carcinoma & 83 & $52.93 \pm 11.28$ \\
\hline Mucinous carcinoma & 7 & $50 \pm 16.33$ \\
\hline Mixed carcinoma & 4 & $50 \pm 7.65$ \\
\hline Total & 196 & $46.26 \pm 14.71$ \\
\hline * mean \pm standard deviation; & & \\
\hline
\end{tabular}


Table 2

Clinical and pathological data summaries in both training and testing cohort.

\begin{tabular}{|c|c|c|c|c|}
\hline & & Training group & Testing group & $P$ value \\
\hline & & $(\mathrm{N}=99)$ & $(\mathrm{N}=97)$ & \\
\hline \multirow[t]{4}{*}{ Age(yrs.) } & & $45.9 \pm 13.35$ & $46.64 \pm 15.90$ & 0.961 \\
\hline & $<30$ & $17(17.2 \%)$ & $14(14.4 \%)$ & \\
\hline & $30-50$ & $35(35.4 \%)$ & $40(41.2 \%)$ & \\
\hline & $>50$ & $47(47.5 \%)$ & $43(44.3 \%)$ & \\
\hline \multirow[t]{4}{*}{ Ki-67 expression (\%) } & & $32.37 \pm 28.01$ & $25.05 \pm 26.35$ & 0.946 \\
\hline & $<50$ & $59(67.0 \%)$ & $74(84.1 \%)$ & \\
\hline & $50-75$ & $20(22.7 \%)$ & $5(5.7 \%)$ & \\
\hline & $>75$ & $9(10.2 \%)$ & $9(10.2 \%)$ & \\
\hline \multirow[t]{5}{*}{ CA-125 level(IU/L) } & & $553.32 \pm 994.28$ & $300.30 \pm 452.27$ & 0.000 \\
\hline & $<35$ & $15(22.7 \%)$ & $18(27.7 \%)$ & \\
\hline & $35-200$ & $17(25.8 \%)$ & $24(36.9 \%)$ & \\
\hline & $200-500$ & $13(19.7 \%)$ & $10(15.4 \%)$ & \\
\hline & $>500$ & $21(31.8 \%)$ & $13(20.0 \%)$ & \\
\hline \multirow[t]{9}{*}{ Category } & & & & 0.980 \\
\hline & Borderline tumor & $47(47.5 \%)$ & $44(45.4 \%)$ & \\
\hline & Malignancies & $52(52.5 \%)$ & $53(54.6 \%)$ & \\
\hline & Endometroid cancer & $2(2.0 \%)$ & $1(1.0 \%)$ & \\
\hline & Low-grade adenocarcinoma & $0(0.0 \%)$ & $3(3.1 \%)$ & \\
\hline & Clear cell type & $1(1 . \%)$ & $4(4.1 \%)$ & \\
\hline & Serous carcinoma & $45(45.5 \%)$ & $38(39.2 \%)$ & \\
\hline & Mucinous carcinoma & $2(2.0 \%)$ & $5(5.2 \%)$ & \\
\hline & Mixed carcinoma & $2(2.0 \%)$ & $2(2.1 \%)$ & \\
\hline
\end{tabular}

\section{Identification results based on MRI-radiomics signatures}

The radiomics signature was weighted with the regression coefficients for the signature construction presented in the form of a histogram in Figure 2. A Rad-score system was calculated using the 
specialized formula after feature selection (Supplementary Table 2). Overall, there was a statistically significant difference observed in the average Rad-score between BOTs (Figure 3) and malignancies in each of the selected MR-based radiomics models $(p<0.0001$, Table 3$)$. Table 4 illustrates the final classification results of the training data set and the validation data set. The model was first determined on the training data set based on the area under the ROC curve (AUC). Then, we evaluated the model on the validation data set. The coronal MR-based radiomics segmentation model yielded an ACC of $78.9 \%$ to $82.8 \%$, while the sagittal model yielded an ACC of $77.8 \%$ to $100 \%$. The 3D sagittal MR-based radiomics model yielded an ACC and an AUC of as high as $100 \%$ in differentiating between BOTs and malignancies in the validation data set (Table 4).

\section{Comparison of the performance results between the 2D and $3 D$ radiomics models}

Considering two acquisition protocols, both coronal and sagittal MR-based features showed competitive accuracy in discriminating BOTs from malignancies either in 2D or 3D segmentation mode (2D AUC: 0.82 versus 0.84 and 3D AUC: 0.79 versus 1.0 , respectively). 3D sagittal fs-T2W images have the best performance compared to the other three methods in discriminating malignancies from BOTs, with an accuracy of $99 \%$ in the testing model. The ROC curve analysis with four kinds of segmentation methods in the validation group is summarized in Figure 4.

Table 3

The average Rad-score between BOT and malignancies in various MR-based radiomics models.

\begin{tabular}{|llll|}
\hline Model & BOT* & $M$ & P value \\
\hline 2D Coronal Training & $-0.73 \pm 0.88$ & $0.63 \pm 0.58$ & $<0.0001$ \\
\hline 2D Sagittal Training & $-0.63 \pm 0.78$ & $0.75 \pm 0.93$ & $<0.0001$ \\
\hline 3D Coronal Training & $-0.74 \pm 0.66$ & $0.87 \pm 1.34$ & $<0.0001$ \\
\hline 3D Sagittal Training & $-8.94 \pm 2.15$ & $9.55 \pm 2.4$ & $<0.0001$ \\
\hline 2D Coronal Testing & $-61.3 \pm 295.1$ & $0.22 \pm 0.90$ & $<0.0001$ \\
\hline 2D Sagittal Testing & $-0.19 \pm 3.28$ & $0.38 \pm 2.99$ & $<0.0001$ \\
\hline 3D Coronal Testing & $-0.66 \pm 0.76$ & $1.14 \pm 1.41$ & $<0.0001$ \\
\hline 3D Sagittal Testing & $-9.16 \pm 2.65$ & $8.89 \pm 2.47$ & $<0.0001$ \\
\hline * mean \pm sd & & & \\
\hline
\end{tabular}


Table 4

The diagnostic performance in differentiating malignancies from BOT based on various MR-based radiomics models.

\begin{tabular}{|llllllll|}
\hline Model & Group & SEN & SPE & PPV & NPV & ACC & AUC(95\% CI) \\
\hline 2d_cor & Training & 0.708 & 0.936 & 0.919 & 0.759 & 0.821 & $0.90(0.85-0.96)$ \\
\hline 2d_cor & Testing & 0.729 & 0.851 & 0.833 & 0.755 & 0.789 & $0.82(0.73-0.90)$ \\
\hline 3d_cor & Training & 0.875 & 0.717 & 0.764 & 0.846 & 0.798 & $0.85(0.77-0.93)$ \\
\hline 3d_cor & Testing & 0.936 & 0.717 & 0.772 & 0.917 & 0.828 & $0.84(0.76-0.93)$ \\
\hline 2d_sag & Training & 0.776 & 0.902 & 0.884 & 0.807 & 0.840 & $0.89(0.83-0.96)$ \\
\hline 2d_sag & Testing & 0.729 & 0.824 & 0.795 & 0.764 & 0.778 & $0.79(0.69-0.88)$ \\
\hline 3d_sag & Training & 1.000 & 1.000 & 1.000 & 1.000 & 1.000 & $1.0(1.0-1.0)$ \\
\hline 3d_sag & Testing & 1.000 & 0.980 & 0.980 & 1.000 & 0.990 & $1.0(1.0-1.0)$ \\
\hline SEN= sensitivity, SPE=specificity, PPV=positive predictive value, NPV=negative positive value, \\
ACC=accuracy, AUC= area under the curve, Cl= confidence interval
\end{tabular}

\section{Discussion}

Ovarian BOT is a type of low-potential epithelial tumor with a relatively good prognosis after treatment. Sometimes, it is difficult to discriminate BOTs from ovarian malignancies solely on imaging information due to some overlapping imaging findings between the two(22). Our current results showed that the 3D MR-based radiomics signatures derived from sagittal fs-T2WI yielded an ACC of $100 \%$ in differentiating ovarian malignancies from BOTs and may help clinicians make a correct diagnosis before surgery. To the best of our knowledge, this is the first reported study focusing on the diagnostic performance of MRbased radiomics signatures in ovarian tumor classification with 2D and 3D segmentation methods.

In the present study, the 3D signatures showed better performance than the 2D signatures did. This result can be easily appreciated because the 3D model utilized information of the whole lesion, more truly reflecting the tumoral heterogeneity than the $2 \mathrm{D}$ model did. The current result is contrary to the previous CT radiomics study in which 2D radiomics features performed slightly better in non-small cell lung cancer prognostic estimation than 3D did (23). The authors concluded that the reason might be related to the various axial CT image resolutions in their study in which the training and validation cohorts in the study sample were selected from different institutions.

Considering the two selected MRI protocols, the fs-sagittal sequence performed better than coronal sequence did on both 2D and 3D segmentation methods. Of note, the 3D-sagittal MR radiomics model yielded ACCs of $100 \%$ and $99 \%$ in the training and testing groups, respectively. This finding is in accordance with our previous study in which fs-T2WI was also superior to coronal T2WI in Type I and Type II ovarian cancer categorization(5). We believe that the sharp contrast between the lesion and the 
background on the fs MR sequence may play a role in the final determination. However, the true mechanism is unclear, and this result should also be validated in a future study with a large study sample.

Several radiomics studies using CT images have been reported for ovarian mass classification and prognostic estimation (24-27). Fathi et al. found that the time-to-peak and wash-in rate parameters showed a high SEN (89\% for the linear discriminant analysis [LDA] classifier and $97 \%$ for the support vector machine [SVM] classifier) and a high SPE (93\% for LDA and 100\% for SVM) in distinguishing malignancies from benign ovarian conditions among 55 sonographically indeterminate ovarian masses (26). Qiu et al. acquired two sets of CT images (pretreatment and posttreatment) to compare three image features (tumor volume, tumor density, and density variance) between the two image sets in 30 ovarian cancer patients, and their model achieved an area under the curve of 0.831 in predicting progression-free survival when combining all three features together(25). In this study, we used the LASSO method to establish the radiomics features model during the radiomics signature selection step as well as during the machine learning process. The Lasso model is reportedly a suitable method for analyzing a small sample with high-dimensional features due to its advantage of avoiding overfitting. A similar method was also reported in two recently published studies with promising results $(18,28)$.

There remains a limited number of studies on MR-based radiomics in ovarian tumor classification and posttreatment response prediction. In one study with 22 patients with advanced ovarian cancer, the authors found that apparent diffusion coefficient (ADC) values derived on the ADC map between primary ovarian cancer and metastatic sites differed significantly and may be used as response markers (29). In the present study, we did not include DW images in the texture analysis. The lesion resolution on DWI, especially with large lesions, is relatively low, which is sometimes difficult to precisely outline in postprocess software. Moreover, in our previous study, we did not find that the ADC map could contribute more useful signatures in task classification than conventional MR images (T1W and T2W images) could (5). Compared with traditional MRI analysis in differentiating BOTs from malignancies, radiomics signature results show better performance. In a traditional MRI reading session, the imaging signs always overlap with each other to some extent (for example, large size, solid components, irregular and thick septa) and lead to an inaccurate diagnosis(27, 30-32). A recent study with proton MRS reported that the SEN and SPE were $91 \%$ and $100 \%$ for solid components, respectively; additionally, the SEN and SPE were $84 \%$ and $82 \%$ for cystic components, respectively (12). However, MRS scans are highly unit-dependent and time-consuming examinations and require operators with more experience than conventional methods do. From this point of view, radiomics signature analysis shows the potential clinical application owing to its simple segmentation step.

The limitations of this study included the fact that we did not include contrast-enhanced (CE) MR images to establish the MRI radiomics model. The CE-MRI scan was not available for all included patients in the current study, and therefore, we did not select this protocol for analysis to diminish the selection bias. Furthermore, in the present study, we only used conventional T2WI to establish a radiomics diagnostic model, which is different from the clinical reading scenario (mostly including T1WI, T2WI and DWI). 
Further study is necessary to explore the difference between one acquisition sequence and multiple acquisition sequences as in the clinical setting. In addition, all segmentation procedures were manually outlined on T2WI showing the best of the lesion; however, it is still an operator-dependent procedure, and interoperator variation in segmentations may be emphasized, especially with multiple sequence images. Finally, all MR images were acquired in a 1.5-T MRI scanner, and a comparison study between 1.5-T and 3.0-T MRI machines should be validated in a large study in the future.

\section{Conclusions}

In summary, our results suggest that radiomics features that were extracted from $\mathrm{T} 2 \mathrm{~W}$ images were highly correlated with ovarian tumor subtype classification. 3D fs-sagittal MRI radiomics features may help clinicians differentiate ovarian BOTs from malignancies with high ACC.

\section{Abbreviations}

BOT Borderline tumor

2D Two-dimensional

3D Three-dimensional

DWI Diffusion weighted imaging

ADC Apparent diffusion coefficient

PACS Picture archiving and communication system

LASSO Least absolute shrinkage and selection operator

LDA Linear discriminant analysis

SVM Support vector machine

\section{Declarations}

\section{Ethics approval and consent to participate}

Our institutional review board approved the study, and the requirement for the informed consent of all participants was waived. In this article, institutional review board was review board of obstetrics and gynecological hospital, Medical College, Fudan University.

\section{Consent for publication}


Not applicable.

\section{Availability of data and materials}

The authors declare that all data supporting the findings of this study are available within the article.

\section{Competing interests}

The authors declare that they have no competing interests.

\section{Authors' contributions}

1. Zhang, K. Hua and G. Zhang designed of the whole study; H. Zhang and S. Duan performed the experiments and analyzed the data; X. Liu performed MRI scan and collected raw data; S.Duan analyzed the data and conducted statistical analysis; J. Jin provides the pathological data; X. Liu and $\mathrm{T}$. Wang revised and wrote the manuscript finally.

\section{Acknowledgements}

Not applicable.

\section{References}

1. Fang C, Zhao L, Chen X, Yu A, Xia L, Zhang P. The impact of clinicopathologic and surgical factors on relapse and pregnancy in young patients ( $\leq 40$ years old) with borderline ovarian tumors. BMC cancer. 2018;18(1):1147-.

2. Hauptmann S, Friedrich K, Redline R, Avril S. Ovarian borderline tumors in the 2014 WHO classification: evolving concepts and diagnostic criteria. Virchows Archiv : an international journal of pathology. 2017;470(2):125-42.

3. Prahm KP, Karlsen MA, Høgdall E, Scheller NM, Lundvall L, Nedergaard L, et al. The prognostic value of dividing epithelial ovarian cancer into type I and type II tumors based on pathologic characteristics. Gynecologic Oncology. 2015;136(2):205-11.

4. Javadi S, Ganeshan DM, Qayyum A, lyer RB, Bhosale P. Ovarian Cancer, the Revised FIGO Staging System, and the Role of Imaging. American Journal of Roentgenology. 2016;206(6):1351-60.

5. Zhang H, Mao Y, Chen X, Wu G, Liu X, Zhang P, et al. Magnetic resonance imaging radiomics in categorizing ovarian masses and predicting clinical outcome: a preliminary study. European Radiology. 2019. Apr 8. doi: 10.1007/s00330-019-06124-9. 
6. Zhao SH, Qiang JW, Zhang GF, Ma FH, Cai SQ, Li HM, et al. Diffusion-weighted MR imaging for differentiating borderline from malignant epithelial tumours of the ovary: pathological correlation. European Radiology. 2014;24(9):2292-9.

7. Li YA, Qiang JW, Ma FH, Li HM, Zhao SH. MRI features and score for differentiating borderline from malignant epithelial ovarian tumors. European Journal of Radiology. 2018;98:136-42.

8. Thomassin-Naggara I, Daraï E, Cuenod CA, Rouzier R, Callard P, Bazot M. Dynamic contrast-enhanced magnetic resonance imaging: A useful tool for characterizing ovarian epithelial tumors. J Magn Reson Imaging. 2008;28(1):111-20.

9. Thomassin-Naggara I, Balvay D, Aubert E, Daraï E, Rouzier R, Cuenod CA, et al. Quantitative dynamic contrast-enhanced MR imaging analysis of complex adnexal masses: a preliminary study. European Radiology. 2012;22(4):738-45.

10. Li H-m, Feng F, Qiang J-w, Zhang G-f, Zhao S-h, Ma F-h, et al. Quantitative dynamic contrastenhanced MR imaging for differentiating benign, borderline, and malignant ovarian tumors. Abdominal Radiology. 2018;43(11):3132-41.

11. Li HM ZS, Qiang JW, Zhang GF, Feng F, Ma FH, Li YA, Gu WY. Diffusion kurtosis imaging for differentiating borderline from malignant epithelial ovarian tumors: A correlation with Ki-67 expression. J Magn Reson Imaging. 2017;46(5):1499-506.

12. Ma FH, Li YA, Liu J, Li HM, Zhang GF, Qiang JW. Role of proton MR spectroscopy in the differentiation of borderline from malignant epithelial ovarian tumors: A preliminary study. Journal of Magnetic Resonance Imaging.0(0).

13. Denewar FA, Takeuchi M, Urano M, Kamishima Y, Kawai T, Takahashi N, et al. Multiparametric MRI for differentiation of borderline ovarian tumors from stage I malignant epithelial ovarian tumors using multivariate logistic regression analysis. European journal of radiology. 2017;91:116-23.

14. Shen D, Wu G, Zhang D, Suzuki K, Wang F, Yan P. Machine learning in medical imaging. Computerized Medical Imaging and Graphics. 2015;41(Supplement C):1-2.

15. Gillies RJ, Kinahan PE, Hricak H. Radiomics: Images Are More than Pictures, They Are Data. Radiology. 2016;278(2):563-77.

16. Gerges L, Popiolek D, Rosenkrantz AB. Explorative Investigation of Whole-Lesion Histogram MRI Metrics for Differentiating Uterine Leiomyomas and Leiomyosarcomas. American Journal of Roentgenology. 2018;210(5):1172-7.

17. Guo C-G, Ren S, Chen X, Wang Q-D, Xiao W-B, Zhang J-F, et al. Pancreatic neuroendocrine tumor: prediction of the tumor grade using magnetic resonance imaging findings and texture analysis with 3-T magnetic resonance. Cancer management and research. 2019;11:1933-44.

18. Zhang B, Tian J, Dong D, Gu D, Dong Y, Zhang L, et al. Radiomics Features of Multiparametric MRI as Novel Prognostic Factors in Advanced Nasopharyngeal Carcinoma. Clinical Cancer Research. 2017;23(15):4259-69.

19. Nougaret S, Tardieu M, Vargas HA, Reinhold C, Vande Perre S, Bonanno N, et al. Ovarian cancer: An update on imaging in the era of radiomics. Diagnostic and Interventional Imaging. 2018. 
20. Altazi BA, Fernandez DC, Zhang GG, Hawkins S, Naqvi SM, Kim Y, et al. Investigating multi-radiomic models for enhancing prediction power of cervical cancer treatment outcomes. Physica Medica: European Journal of Medical Physics. 2018;46:180-8.

21. Zhang B, Ouyang F, Gu D, Dong Y, Zhang L, Mo X, et al. Advanced nasopharyngeal carcinoma: pretreatment prediction of progression based on multi-parametric MRI radiomics. Oncotarget. 2017;8(42):72457-65.

22. Liu D, Zhang L, Indima N, Peng K, Li Q, Hua T, et al. CT and MRI findings of type I and type II epithelial ovarian cancer. European journal of radiology. 2017;90:225-33.

23. Shen C, Liu Z, Guan M, Song J, Lian Y, Wang S, et al. 2D and 3D CT Radiomics Features Prognostic Performance Comparison in Non-Small Cell Lung Cancer. Translational oncology. 2017;10(6):886-94.

24. Rizzo S, Botta F, Raimondi S, Origgi D, Buscarino V, Colarieti A, et al. Radiomics of high-grade serous ovarian cancer: association between quantitative CT features, residual tumour and disease progression within 12 months. European Radiology. 2018.

25. Qiu Y, Tan M, McMeekin S, Thai T, Ding K, Moore K, et al. Early prediction of clinical benefit of treating ovarian cancer using quantitative CT image feature analysis. Acta radiologica (Stockholm, Sweden : 1987). 2016;57(9):1149-55.

26. Fathi KA, Mahrooz M, Hamidreza H, Sara P, Mahnaz N, Leila T, et al. Semiquantitative dynamic contrast-enhanced MRI for accurate classification of complex adnexal masses. Journal of Magnetic Resonance Imaging. 2017;45(2):418-27.

27. Vargas HA, Miccò M, Hong SI, Goldman DA, Dao F, Weigelt B, et al. Association between Morphologic CT Imaging Traits and Prognostically Relevant Gene Signatures in Women with High-Grade Serous Ovarian Cancer: A Hypothesis-generating Study. Radiology. 2015;274(3):742-51.

28. Vasquez MM, Hu C, Roe DJ, Chen Z, Halonen M, Guerra S. Least absolute shrinkage and selection operator type methods for the identification of serum biomarkers of overweight and obesity: simulation and application. BMC Medical Research Methodology. 2016;16:154.

29. Sala E, Kataoka MY, Priest AN, Gill AB, McLean MA, Joubert I, et al. Advanced Ovarian Cancer: Multiparametric MR Imaging Demonstrates Response- and Metastasis-specific Effects. Radiology. 2012;263(1):149-59.

30. Feng W, Yuxiang W, Yan Z, Congrong L, Lizhi X, Zhenyu Z, et al. Comparison between types I and II epithelial ovarian cancer using histogram analysis of monoexponential, biexponential, and stretchedexponential diffusion models. Journal of Magnetic Resonance Imaging. 2017;46(6):1797-809.

31. Lalwani N, Prasad SR, Vikram R, Shanbhogue AK, Huettner PC, Fasih N. Histologic, Molecular, and Cytogenetic Features of Ovarian Cancers: Implications for Diagnosis and Treatment. RadioGraphics. 2011;31(3):625-46.

32. Sahdev A. CT in ovarian cancer staging: how to review and report with emphasis on abdominal and pelvic disease for surgical planning. Cancer Imaging. 2016;16:19.

\section{Figures}



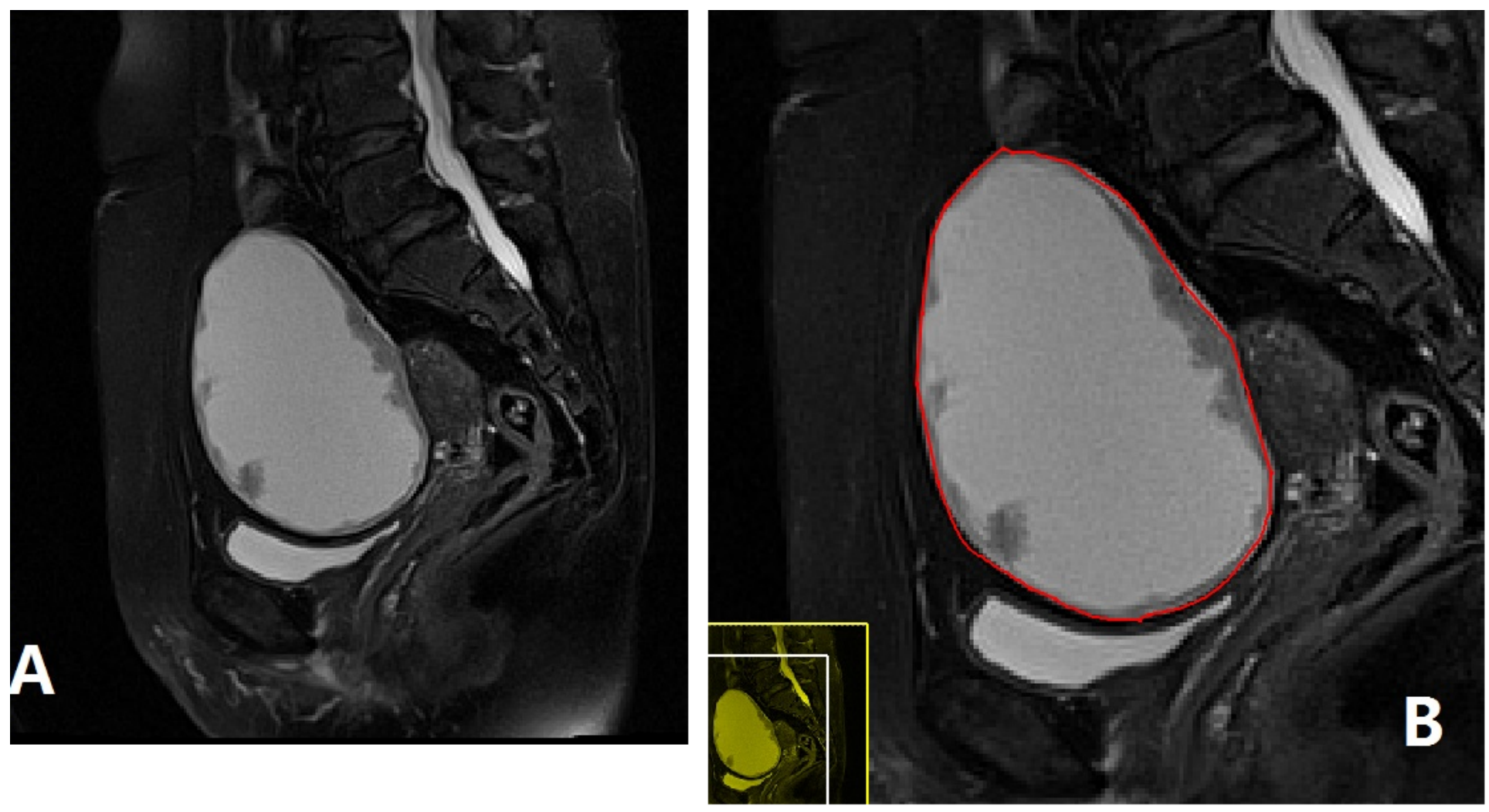

Figure 1

MR-based radiomics signatures were extracted from 2D/3D sagittal fs-T2W and 2D/3D coronal T2W images using Analysis Kit software( version 3.0.0, GE Healthcare) on a personal computer 


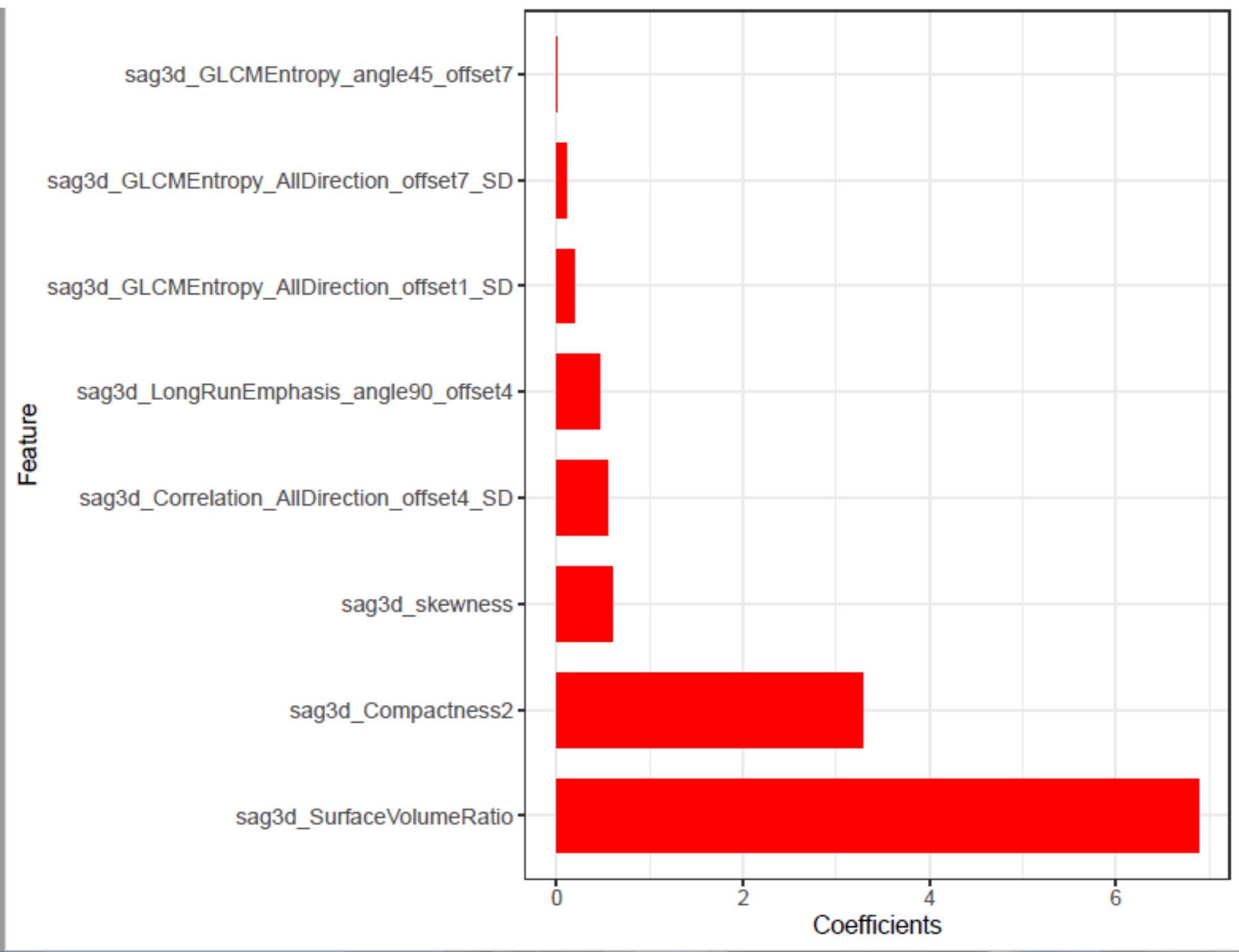

Figure 2

The radiomics signature was weighted with the regression coefficients for the signature construction presented in the form of a histogram 

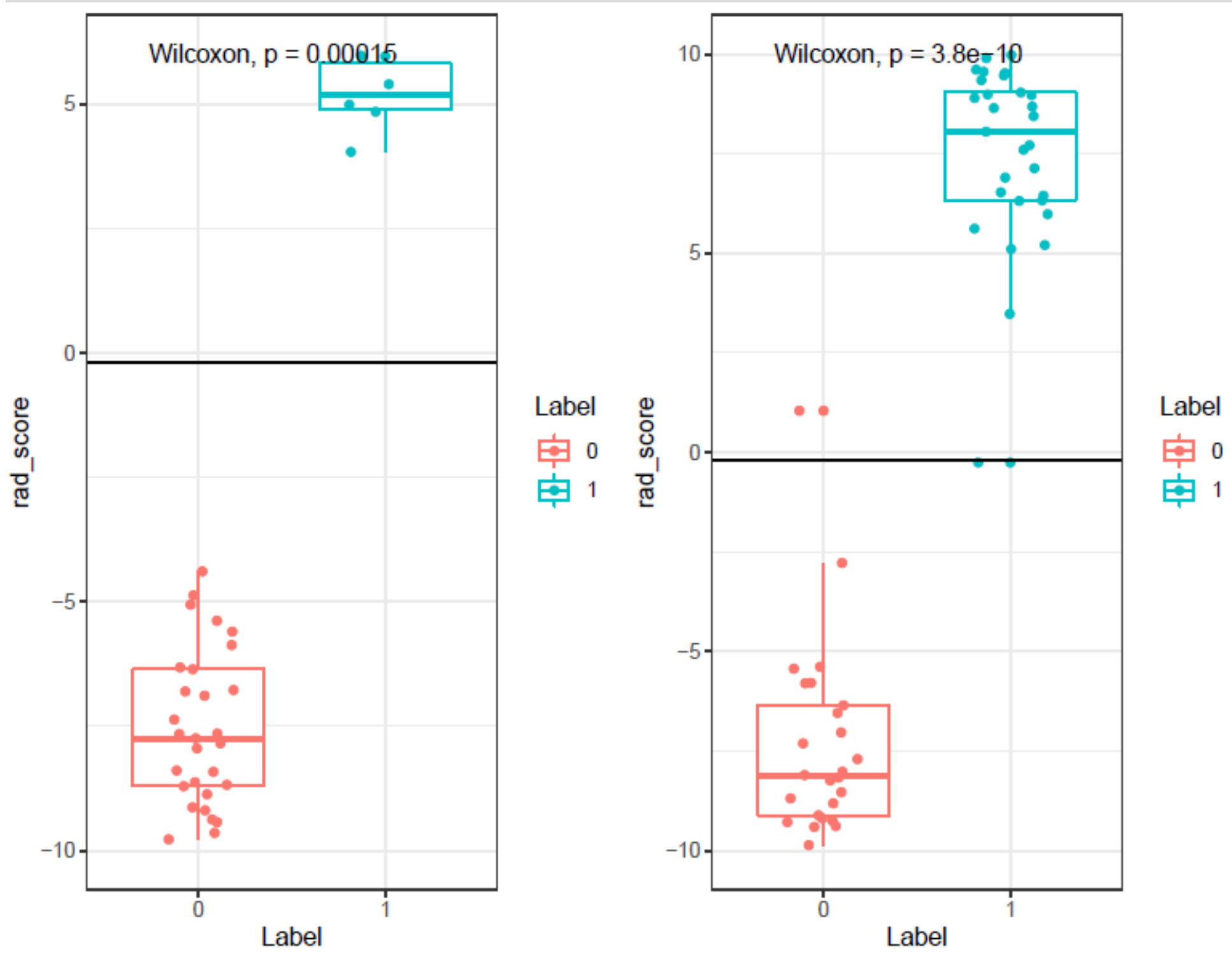

Figure 3

statistically significant difference observed in the average Rad-score between BOTs 


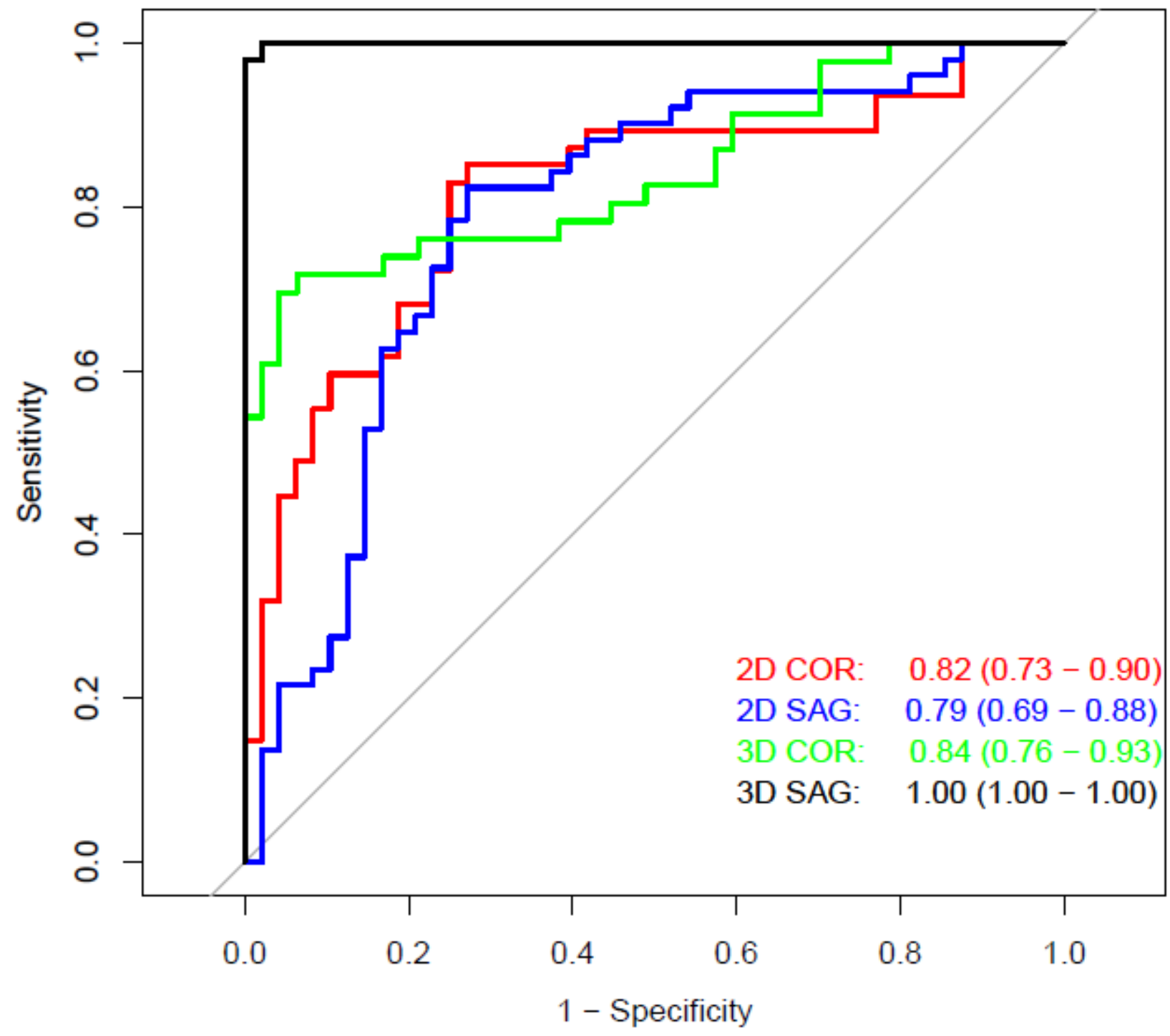

Figure 4

The ROC curve analysis with four kinds of segmentation methods in the validation group is summarized 